\title{
Performance Analysis of Statistical QoS Routing Schemes for Multihop Wireless Ad Hoc Networks
}

\author{
Mitko Bogdanoski ${ }^{1}$, Dimitar Bogatinov ${ }^{1}$ and Saso Gelev ${ }^{2}$ \\ ${ }^{1}$ Militar Academy "General Mihailo Apostolski", an associated member of Goce \\ Delcev University \\ ${ }^{2}$ Goce Delcev University \\ \{mitko.bogdanoski,dimitar.bogatinov,saso.gelev\}@ugd.edu.mk
}

\begin{abstract}
The quality of service $(Q o S)$ standard in Mobile Ad-hoc Networks (MANET's) is much more challenging compared to the wired networks. In the last decade there was a lot of attention in the area of integration of QoS standard in MANET protocols. QoS-based routing protocols are integral part of any QoS solution, because their function is to check whether the nodes in the network are able to provide services to network applications. These protocols play a key role in controlling the information received and delivered from data transfer sessions. The purpose of this paper is to study and review the QoS routing schemes designed for IEEE 802.11 standard in Multihop Ad-hoc networks. Moreover, the paper simulates a routing scheme and shows results for an effective end-to-end communication.
\end{abstract}

Keywords: Mobile ad-hoc networks (MANETs), IEEE 802.11 standard, QoS routing, routing protocols

\section{Introduction}

Communication networks are backbone for operation of any successful company/organization. The role of these networks is to enable end to end communication and to transport a multitude of applications and data, including high-quality video and delaysensitive data such as real-time voice. The bandwidth-intensive applications stretch network capabilities and resources, but also complement, add value, and enhance every business process. Networks must provide secure, predictable, measurable, and sometimes guaranteed services. The purpose of the QoS standard is to provide preferential delivery services that need ensuring sufficient bandwidth, controlling latency and jitter, and reducing data loss. $[1,2]$. The Table 1 describes these network's characteristics.

Table 1. Network Characteristics

\begin{tabular}{|l|l|}
\hline Bandwidth & The delivered amount of information per time unit \\
\hline Latency & The delay in handing over information from one node to another \\
\hline Jitter & The variation in latency. \\
\hline Reliability & The percentage of packets discarded by a router. \\
\hline
\end{tabular}

Actually, the QoS guarantee throughput for mission-critical applications so that their transactions can be processed in an acceptable amount of time. Network administrators use 
the QoS standard to control and manage the UDP (User Datagram Protocol) traffic. Unlike TCP (Transmission Control Protocol), UDP is a protocol that does not receive feedback from the network, ie, it cares only about packets' forwarding and cannot detect network overload. The QoS standard can also be used to control the priority of applications that work with UDP protocol, such as multimedia applications in providing of the necessary width of the frequency range at times when the network is overloaded.

The rest of the paper is organized as following. Section 2 provides an overview in ad-hoc networks with multiple hops. In Section 3 QoS routing techniques in MANET are explained. Moreover, the Section 4 gives explanation about the QoS support based on the bandwidth calculation. Furthermore, the Section 5 gives explanation about the statistical QoS routing scheme. The simulation and results from the simulation are shown in Section 6. The last section conclude the work in this paper.

\section{Multihop ad-hoc Networks}

The wireless mobile communication is based on the infrastructure of the wired network. Mobile devices use one hop radio communication to gain access to the base station which is connected to the wired infrastructure. But, it does not necessarily means that those networks cannot be realized in circumstances where there is no wired infrastructure. Multihop ad-hoc networks (Figure 1) consist of a set of wireless nodes that have limited resources like: bandwidth, memory, power supply, etc. The entire set of wireless links is establishing a wireless Multihop topology in which wireless nodes change their status (active mode operating / idle mode operation) and makes the ad-hoc network topology to be dynamic. There are two basic types of Multihop wireless ad-hoc networks: Mobile ad-hoc networks (MANET) and wireless sensor networks [3-6].

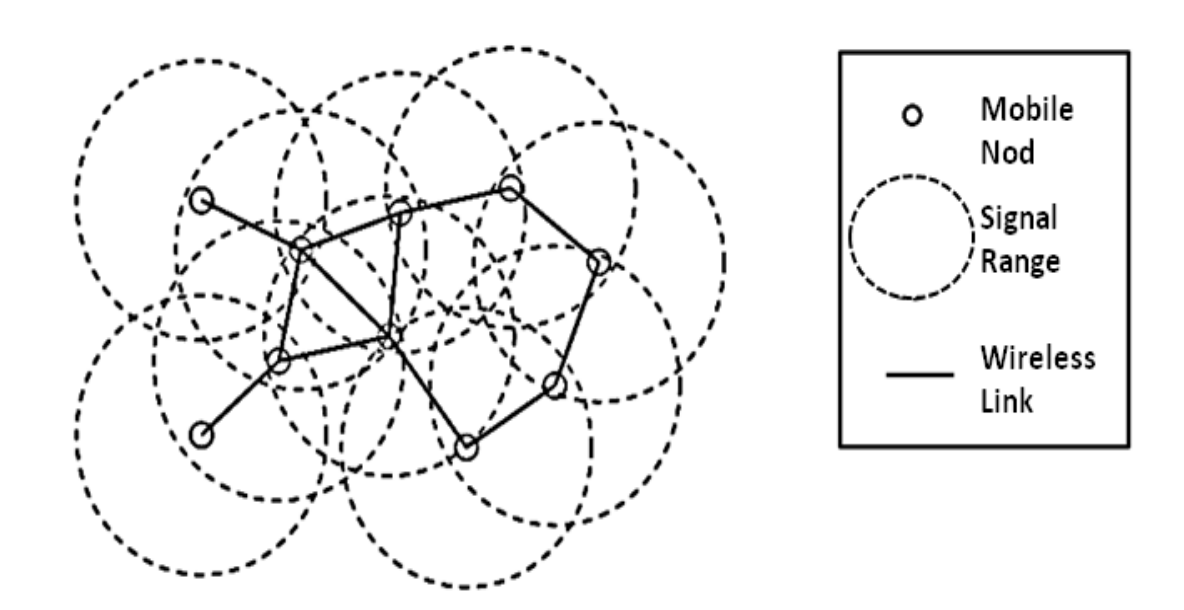

Figure 1. Multihop ad-hoc Network

MANET consists of wireless devices that communicate with each other and do not contain any fixed infrastructure or central administration. In these types of networks, Multihop routing with is necessary when the destination is outside the sender's network coverage. Routing protocols in these networks are divided into two classes: proactive and reactive routing protocols.

In proactive protocols, each node of the network contains information about the routes to all possible destinations, regardless of their use. Unlike this, in reactive protocols the node discovers the route and keeps it only for the necessary time. 


\section{QoS routing in MANET'S}

The QoS routing schemes in MANET can be divided into three categories [3,7]:

- precomputed table-based routing schemes,

- on-demand source-based routing schemes and

- constraint-based routing schemes.

Precomputed routing scheme are based on routing tables, ie, every node of the network includes routing tables that store routing information. The information in the routing tables are used to determine the next hop of the packets to reach the destination. Examples for standards or protocols that can be used for this type of routing are WRP (Wireless Routing Protocol), DSDV (Destination Sequenced Distance Vector) and CGSR (Clusterhead Gateway Switch Routing).

Difference between precomputed routing scheme and on-demand source-based routing schemes are that the source routed protocols work on another principle, creating routes as needed, ie, when a node on the network wants to communicate with another, it initialize a process that requires the route. When the route is found it is maintained until the end of the routing. Standards or protocols that can be used for this type of routing are: AODV (Ad hoc On-demand Distance Vector), DSR (Dyniamic Source Routing), TORA (Temporary Ordered Rouitng Algorithm), GPSR (Greedy Perimeter Stateless Routing). The main focus of these protocols and standards are to find the shortest path between the nodes that need to perform the routing.

Unlike these protocols that are characterized by selecting the shortest route, constraintbased routing schemes have slightly different way of choosing the appropriate routing path. ABR (Associativity Based Routing) and SSR (Signal Stabilty Routing) take into account the node's signal strength and location stability so that the path chosen is more likely to be longlived.

In Figure 2 the wireless nodes in the network are labeled as $\mathrm{A}, \mathrm{B}, \mathrm{C}, \mathrm{D}, \ldots \mathrm{K}$, and the numbers found besides each edge represents the available bandwidth of the wireless links.

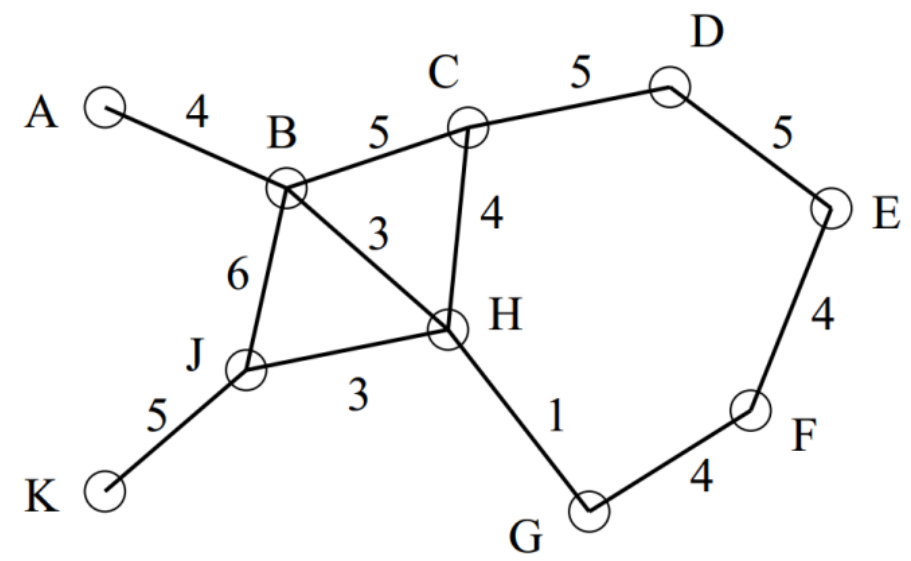

Figure 2. QoS Routing in Ad-hoc Networks

It is assumed that it is necessary to find a route between the node A and node G. Using protocol which is based on the traditional shortest path routing (in terms of number of hops) the route will be A-B-H-G, but if we choose the QoS routing, the routing table would be very different. If we consider the available bandwidth between the nodes, as one of the main features of QoS, with minimum bandwidth 4, then the feasible route will be A-B-C-D-E-F-G. 
Good QoS routing algorithms includes the following characteristics:

- Routing algorithm should calculate an efficient route that satisfies the standard for quality service with a high probability.

- QoS algorithm which calculates the route should be simple and robust.

- If there is a change in network dynamics, the propagation and updates in state information should be kept to a minimum.

\section{QoS Support Using Bandwidth Calculation}

Calculation of the information flow or bandwidth for ad-hoc networks can be done using algorithm with time allocation or TDMA (Time Division Multiple Access) [8]. Using this algorithm, the node that sends information can determine the necessary resources that it needs for supporting QoS to any destinations in ad-hoc network. Working with time slots in ad-hoc networks is a bit more complex than in wired networks, because it needs to know the condition of the free time slots and need to generate a free time slots for each hop. The entire process is illustrated in Figure 3 [9].

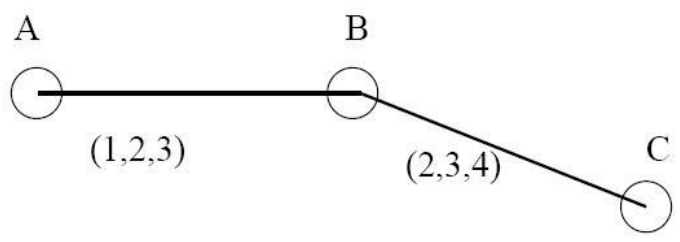

Figure 3. Bandwidth Calculation in Ad-hoc Network

Time slots 1,2,3, between nodes A and B, and time slots 2,3,4, between nodes B and C, are free. Assuming that node $A$ wants to send information to node $C$. It could be noted that collisions will occur at node B if node A use all three slots $(1,2,3)$ to send data to node $\mathrm{B}$, while the node B uses one or both at the same time (2.3) time slots to send data to node C. In TDMA systems, the time is divided into slots which in turns is grouped in frames. Each frame has two phases: a control phase and a data phase. In the control phase, each node of the network in predefined slot broadcast its information to all its neighboring nodes, and by the end of the control phase each node of the network receives information about the free time slots between itself and its neighboring nodes. Based on this information, the calculation of the data flow or bandwidth can be performed distributively.

\section{Statistical QoS Routing Scheme}

When discussing the statistical routing, it is necessary to discuss about the previously mentioned GPSR protocol. GPSR routing protocol uses a technique called (Greedy Packet Forwarding). In this technique, the packets from the sender includes the position of the receiver. When the packets arrives at a non addressed node it checks the packet and forwards it to its neighboring node that is geographically closer to the node that the packet is addressed to. This process is repeated for every hop, until it reaches the desired destination. Figure 4 shows the nodes in the network labeled as A, B, C, D, E. GPSR protocol sends a list with its neighboring nodes to each node of the network with the position and identification (ID) of the neighboring nodes [1]. 


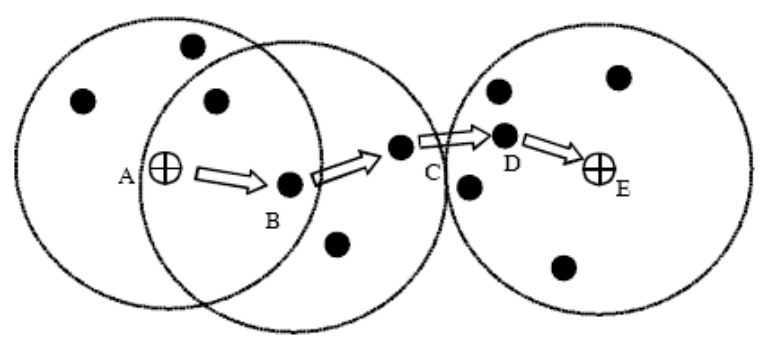

Figure 4. Route Retrieval Process

Node A starts a route discovery by sending RR (Route Request) message to the geographically closest neighboring node with respect to the packet destination. RR message contains information about the position of the destination and information about the traffic. Once found, node A keeps its ID for further forwarding. Node that is found next between the sender and recipient information puts its ID in the RR message and performs the same procedure as node $\mathrm{A}$, ie, RR resends the message and repeat it until the destination node is reached. If we assume that the retrieval process reached node $\mathrm{C}$ and for some reason cannot realize the next hop, then the node $\mathrm{C}$ starts a new route retrieval process, excluding the interrupted hop from its table.

In case when all next hops after the first hop are terminated, this node instead of sending RR message sends RP (Route Repair) message. When the receiving node gets the RP message it starts a process that removes the problem. If a problem occurs with the first hop (node A), then the route retrieval process is renewed.

\section{Simulation and Results}

The simulation is done using OPNET Modeler simulation tool [10-12]. The paper considers 4 scenarios that analyze the characteristics of AODV and DSR routing protocol. The first two scenarios show the characteristics of these two protocols in a rarely populated area with mobile nodes (in our case 4). The next two simulations are representing the characteristics of these two protocols in more dense area (in our case 10).

Firstly we review the simulations in which the areas are rarely populated, with 4 mobile nodes in same IP subnet. The first node in the 100th second generates traffic which exponentially increases until the end of the simulation. The simulation runs 10 minutes. In the 370th second of the simulation, one node changes its location. This situation can be reason for breaking the established route. During the simulation only few parameters that will indicate certain features of the protocols will be considered. The monitored parameters are: the router discovery time (average time during simulation), the total packets dropped, the total routes errors sent and the total route requests sent. The results and conclusions are derived from the obtained graphs.

It should be noted that the both protocols are of the same (on-demand) type; however these protocols differ in certain essential features. DSR routing protocol is a source routing protocol, while AODV is a destination routing protocol. This indicates that the DSR protocol sets the route path in the header of each packet at start, and do not need to store routing parameters in between nodes, while AODV protocol keeps active all the nodes that participate in the transfer, so each node remembers the next node to which it should send a packet, not carrying the information for the entire route. 


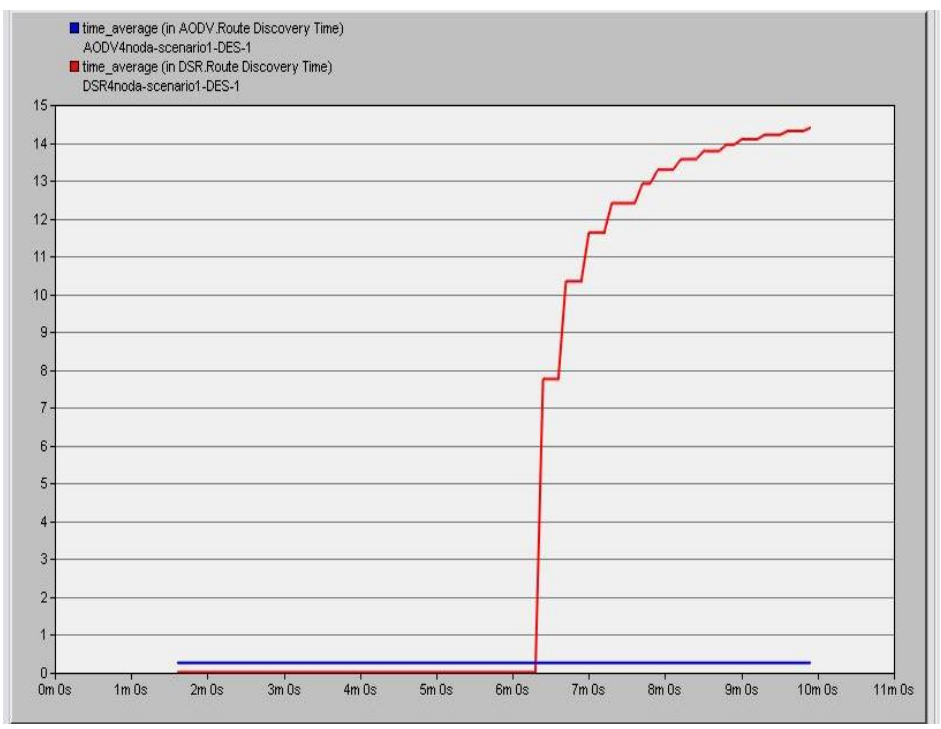

Figure 5. Comparison between AODV and DSR Route Discovery Time (4 nodes)

Figure 5 shows that during the simulation when AODV routing protocol is used there is no lose of time after the first route discovery, while DSR protocol needs time for route discovery at each next node in the path to the destination node. Considering this parameter, the AODV proves to be better routing protocol than DSR routing protocol.

Figure 6 compares the total number of packets dropped. Considering this parameter it can be concluded that DSR routing protocol has worse characteristics than AODV routing protocol. It can be seen that, for the same configuration, there are 205 dropped packets when the DSR routing protocol has been used, and only 185 dropped packets during the usage of AODV.

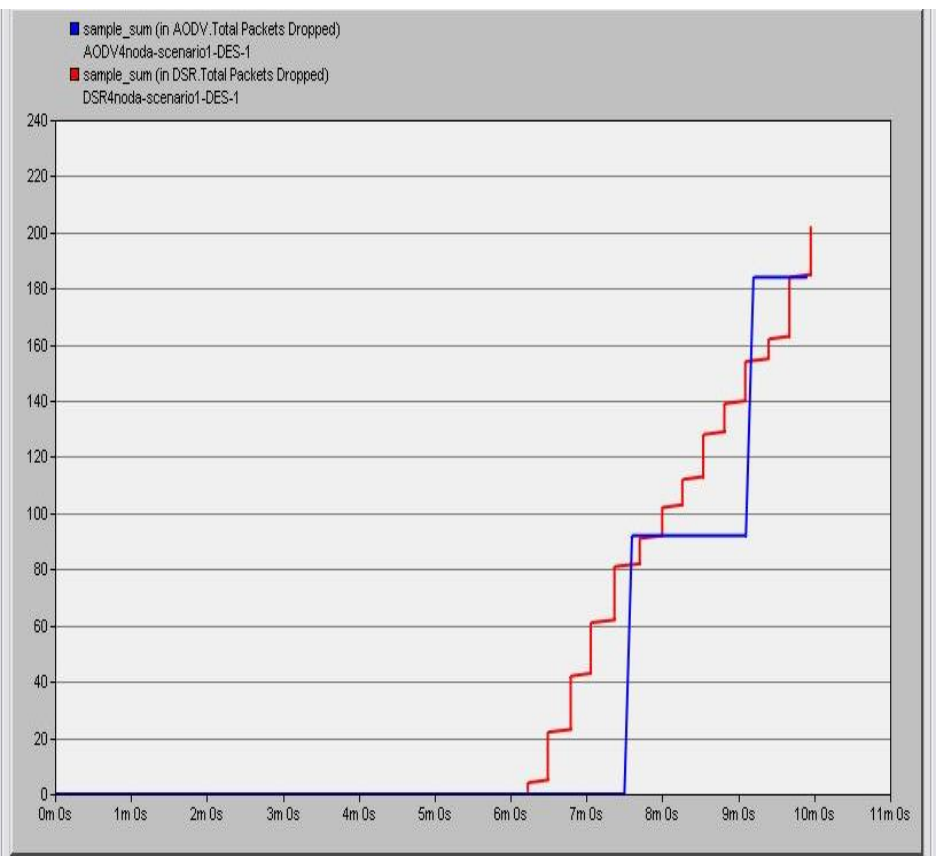

Figure 6. Total Packets Dropped (4 nodes) 
Figure 7 shows the total route errors sent when AODV and DSR protocols are used.

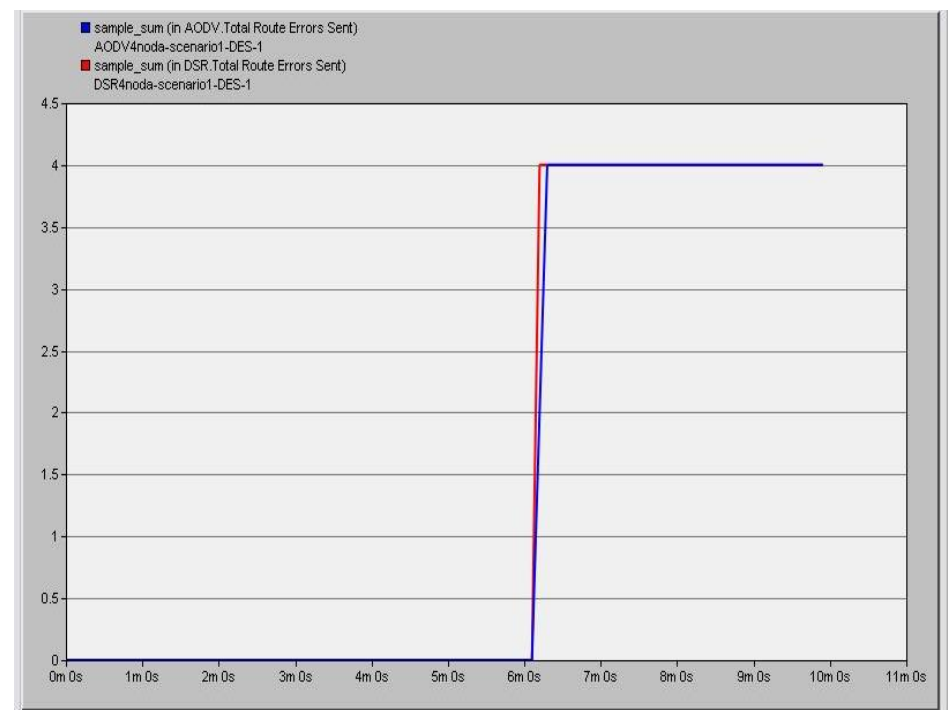

Figure 7. Total Route Errors Sent (4 nodes)

In this segment, both protocols are equally effective.

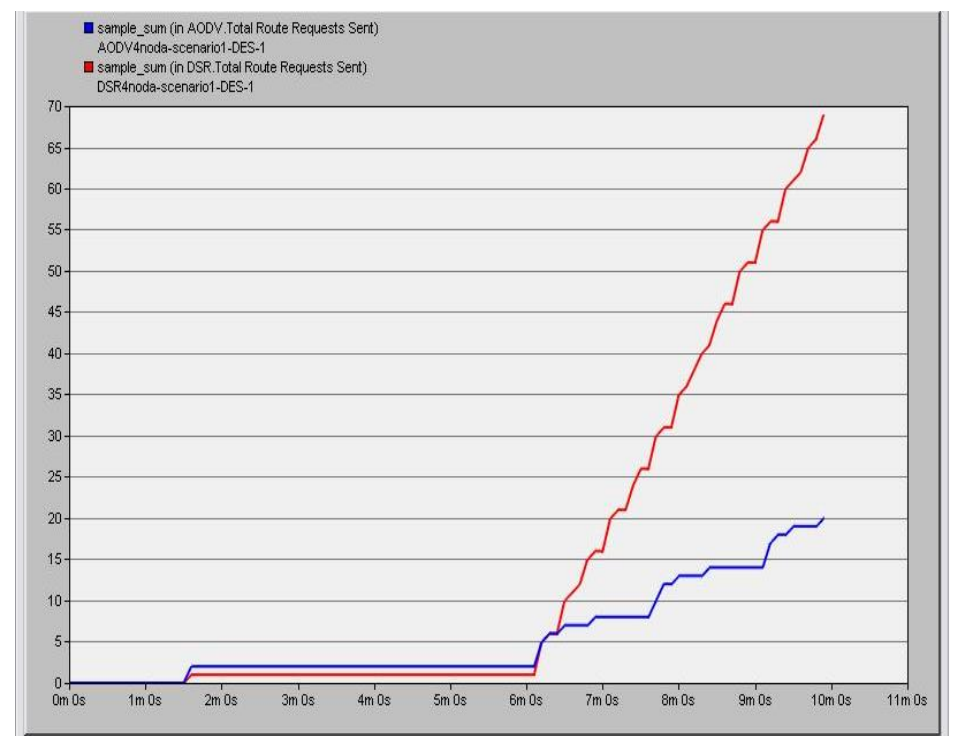

Figure 8. Comparison of the Route Requests Sent (4 nodes)

Figure 8 compare the route requests for both scenarios. Considering the graph it can be concluded that AODV provides better features than the DSR routing protocol.

The following Figures $(9,10$ and 11) shows comparisons of the same routing protocols but in network environment with more nodes. Here we set the same characteristics that apply in previous scenarios and the only difference is that instead of 4 nodes, in the following two scenarios we implemented 10 nodes for the same area. 


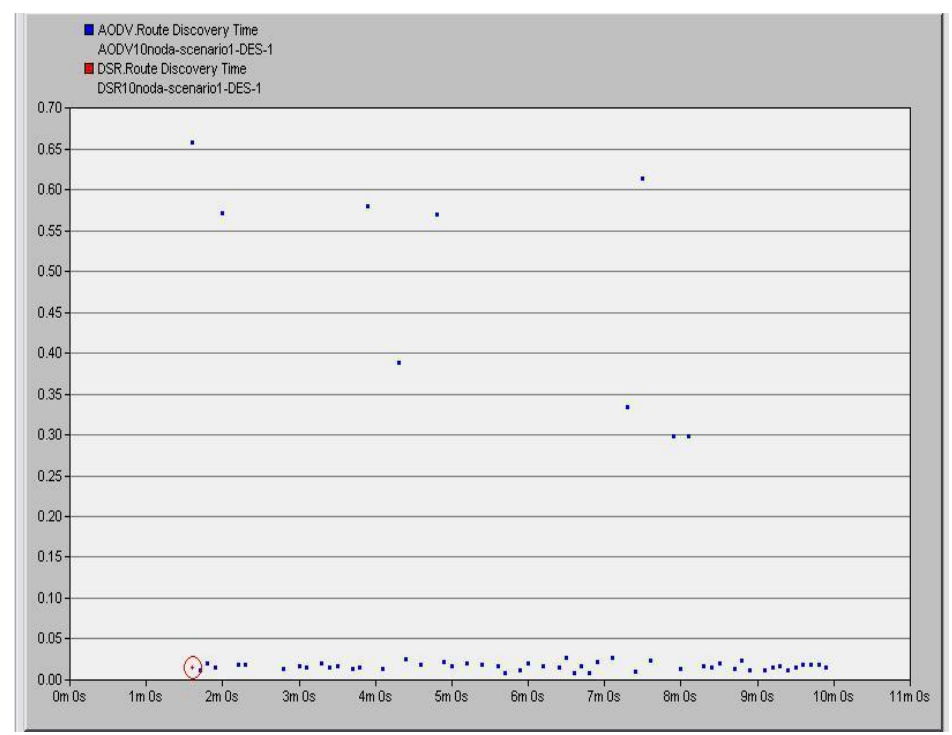

Figure 9. Route Discovery Time (10 nodes)

Figure 9 compares the route discovery time, where DSR gives much better results than AODV routing protocol.

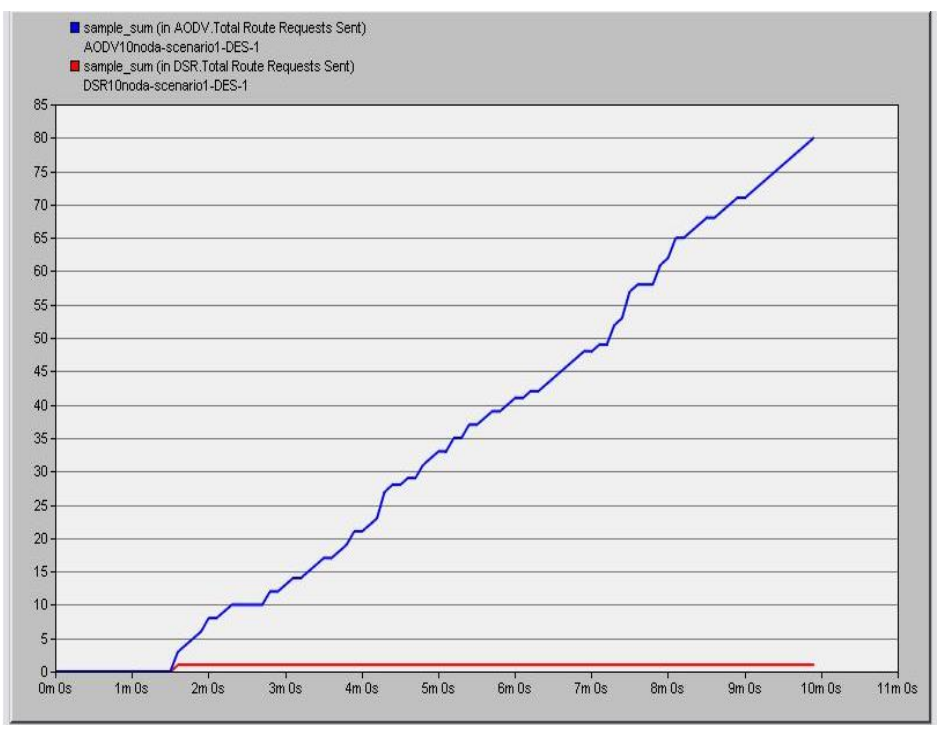

Figure 10. Total Route Requests Sent (10 nodes)

Figure 10 presents the comparison of total route requests sent. In this scenarios DSR gives better results than AODV because DSR protocol has no route requests after the initial request, while AODV requests routes repeatedly while there is a data transfer. 


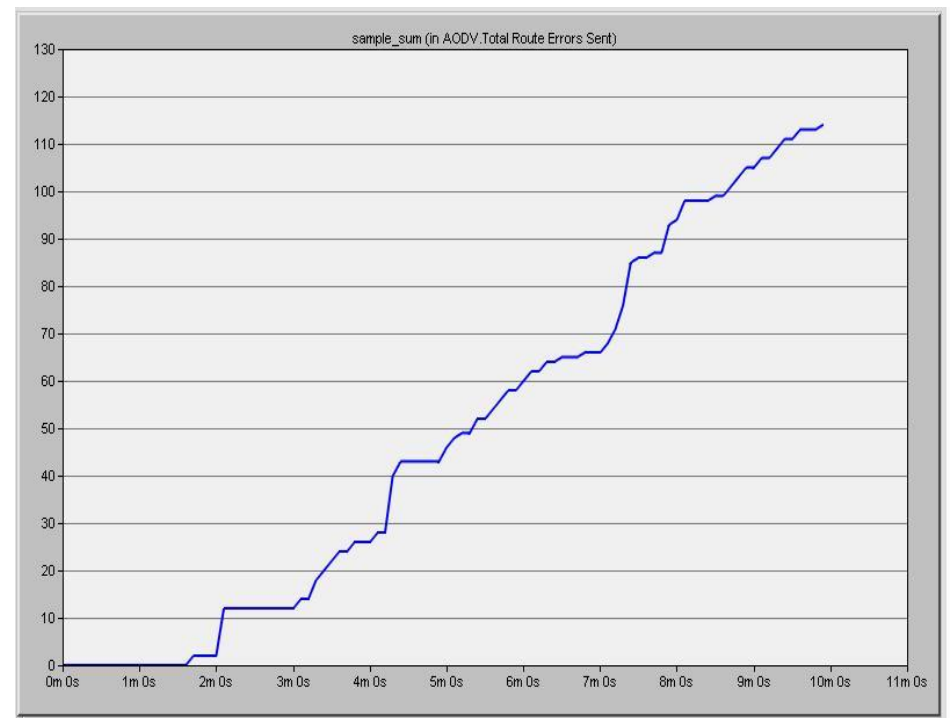

Figure 11. Total Route Errors Sent (10 nodes)

Figure 11 represents the total route errors sent. The DSR protocol has no error of this nature, while AODV-based scenario accumulates errors over the time and their total number is gradually increasing. Considering these results we can freely conclude that in more density networks DSR is shows better performances compared to the AODV routing protocol.

\section{Conclusion}

The paper describes the majority of ad-hoc routing protocols and the features that lead to a QoS-based routing. In the experimental part (simulation) the paper considers only two protocols (AODV and DSR) and it conduct a comparison of how these protocols affect some of the QoS characteristics. Both protocols are on-demand routing protocols, except that one is source routing (DSR), while the other is destination routing (AODV).

From the simulation it can concluded that in an environment with a smaller number of nodes (4 nodes) and low mobility, the AODV protocol gives better results than DSR routing protocol, but in environments with an increased number of nodes (10 nodes) and increased mobility, DSR protocol gives better results than AODV routing protocol.

According to previously mentioned, we can conclude that depending on the simulation parameters we have change of the characteristics and the results of the two protocols.

Therefore, for good quality of services in mobile ad-hoc networks it is necessary to know the network topology, the positioning of the nodes, their mobility and the type of service that they are going to be used for, so that the protocol we chose has the maximum effect in such circumstances.

As future research resulting from this work it is planned to verify the operation and quality of service for AODV and DSR protocols, as well as other WoSObased routing protocols, in an environment where there are extreme density of nodes (over 100 nodes), in an environment where we have greater nodes' mobility, and in circumstances where there are different type of services (different types of generated traffic). 


\section{References}

[1] A. Abdrabou and W. Zhuang, "Statistical QoS Routing for IEEE 802.11 Multihop Ad Hoc Networks", IEEE Transactions on Wireless Communications, vol. 8, no. 3, (2009).

[2] M. Balachandra, K. V. Prema and K. Makkithaya, "Multiconstrained and multipath QoS aware routing protocol for MANETs", Springer, Wireless Networks, (2014).

[3] J. Li, "Quality of Service (QoS) Provisioning in Multihop Ad Hoc Networks", University of California, Davis, (2006).

[4] P. Nicopolitidis, M. S. Obaidat Monmouth, G. I. Papadimitriou and A. S. Pomportsis, "Wireless Networks", Wiley, (2003).

[5] V. K. Garg, "Wireless Communications And Networking", Morgan Kaufmann, (2007).

[6] Z. Mehmood, M. Iqbal and X. Wang, "Comprehensive Experimental Performance Analysis of DSR, AODV and DSDV Routing Protocol for Different Metrics Values with Predefined Constraints", International Journal of Information Technology and Computer Science(IJITCS), July (2014).

[7] S. Biswas and R. Morris, "ExOR: Opportunistic MultiHop Routing for Wireless Networks, SIGCOMM '05", Proceedings of the 2005 conference on Applications, technologies, architectures, and protocols for computer communications, (2005).

[8] C. Lin and J. Liu, "QoS Routing in Ad Hoc Wireless Networks," IEEE Journal on Selected Areas in Communications, (1999).

[9] L. Hanzo (II.) and R. Tafazolli, "A Survey of QoS Routing Solutions for Mobile Ad hoc Networks", IEEE Communications Surveys \& Tutorials, vol. 9, no. 2, (2007), pg. 50-70.

[10] R. Gupta and H. Aggarwal, "Article: Network Density based Analysis of MANET Routing Protocols", International Journal of Computer Applications vol. 77, no. 10, (2013), pp. 33-39, September

[11] B. Mihajlov and M. Bogdanoski, "Overview and Analysis of the Performances of ZigBee based Wireless Sensor Networks" International Journal of Computer Applications, vol. 29, no. 12, pp. 28-35, (2011).

[12] S. V. Nandal and K. V. Sonia, "OPNET based Performance Evaluation of MANET using IEEE 802.11a/b", International Journal of Computer Science and Mobile Computing, vol. 3 no. 6, (2014), pg. 878-886. 